Review

\title{
Use of NMR applications to tackle future food fraud issues
}

\author{
Anatoly P. Sobolev ${ }^{\mathrm{a}}$, Freddy Thomas ${ }^{\mathrm{b}}$, James Donarski ${ }^{\mathrm{c}}$, Cinzia Ingallina ${ }^{\mathrm{d}}$, Simone Circi $^{\mathrm{d}}$, \\ Flaminia Cesare Marincola ${ }^{\mathrm{e}}$, Donatella Capitani ${ }^{\mathrm{a}}$, Luisa Mannina ${ }^{\mathrm{d}, \mathrm{a}, *}$ \\ a Istituto per i Sistemi Biologici, Laboratorio di Risonanza Magnetica “Annalaura Segre", CNR, Monterotondo, Rome, Italy \\ ${ }^{\mathrm{b}}$ Eurofins Analytics France, Rue Pierre Adolphe Bobierre, 44323, Nantes Cedex 3, France \\ ${ }^{\mathrm{c}}$ Fera Science Ltd, Sand Hutton, York, YO41 1LZ, United Kingdom \\ ${ }^{\mathrm{d}}$ Dipartimento di Chimica e Tecnologie del Farmaco, Sapienza Università di Roma, Piazzale Aldo Moro 5, Rome, Italy \\ ${ }^{\mathrm{e}}$ Dipartimento di Scienze Chimiche e Geologiche, Università di Cagliari, Monserrato, Cagliari, Italy
}

\section{A R T I C L E I N F O}

\section{Keywords:}

NMR

Food fraud

Metabolomics

Routine application

Food authenticity

NMR database

\begin{abstract}
A B S T R A C T
Background: NMR targeted and untargeted methodologies are widely recognized as important tools for food authentication and the detection of counterfeit products. Targeted approaches allow the identification of specific markers of identity/adulteration for a given foodstuff. In the untargeted approach, the chemical profile of the whole foodstuff is used to create a unique fingerprint as a reference for suspect samples. The untargeted analysis methodology typically follows the metabolomics approach.

Scope and approach: In this manuscript we discuss how both targeted and untargeted NMR methodologies are applied in routine use for food fraud monitoring. The cost-effective approaches for routine application are discussed using examples of Food Screener ${ }^{\mathrm{TM}}$ and benchtop low-field instruments.

Key findings and conclusions: Several examples of routine consolidated NMR targeted and untargeted applications are reported and the food matrices that are problematic for the NMR application are discussed. The future NMR implementation into routine practice will rely on the further exploration of FoodScreener ${ }^{\mathrm{TM}}$ like platforms for simultaneous targeted and untargeted applications and the continued development of applications for low-field benchtop instrumentation.
\end{abstract}

\section{Introduction}

Nuclear Magnetic Resonance (NMR) methodologies are widely recognized as an important tool for food analysis since they enable the study of both chemical composition and molecular dynamics of solid, liquid and semisolid food matrices (Mannina et al., 2017; Proietti et al., 2017; Sobolev et al., 2017).

In this scientific opinion, a short discussion regarding NMR methodologies and, in particular, the metabolomics approach used in the monitoring of food authenticity is reported. A significant emphasis is given to the implementation of the NMR technology to routine applications. Relevant and up-to-date examples of applications on different food systems are summarized in Table 1 . They include notable contributions by several academic laboratories to the analysis of liquid food by high-resolution NMR, solid insoluble foods by high-resolution magic-angle spinning (HR-MAS) or benchtop NMR and commercial NMR food screener database.

\section{NMR methodologies in food authenticity monitoring}

NMR methodologies can be useful to characterize food matrices in terms of quality and authenticity, allowing also the identification of counterfeits (Sobolev, Circi, \& Mannina, 2016). Basically, this can be accomplished by using one of two approaches, either targeted or untargeted analysis according to specific application.

The targeted approach allows the identification of specific markers of identity/adulteration for a given foodstuff. For example, high resolution ${ }^{1} \mathrm{H}$ NMR analysis was successfully applied to detect the presence of 16-O-methylcafestol (16-OMC) in roasted coffee labeled as " $100 \%$ Arabica". 16-OMC is a chemical marker of less expensive Robusta coffee, therefore its presence in Arabica coffee confirms the adulteration (Monakhova et al., 2015). Another example is the control of sugar addition in honey: the characteristic minor sugar turanose present in honey can be easily observed and quantified by ${ }^{1} \mathrm{H}$ NMR. The absence or extremely low levels of this molecule has been reported to indicate the dilution of honey with sugar syrup (Spiteri et al., 2015).

Using specific markers, the NMR approach has been also used for

\footnotetext{
* Corresponding author. Dipartimento di Chimica e Tecnologie del Farmaco, Sapienza Università di Roma, Piazzale Aldo Moro 5, Rome, Italy.

E-mail address: luisa.mannina@uniroma1.it (L. Mannina).
} 
Table 1

Relevant and up-to-date examples of NMR applications on food systems.

\begin{tabular}{|c|c|c|c|}
\hline NMR technique & Food Matrix & Determination & References \\
\hline \multirow[t]{17}{*}{ High Resolution NMR } & \multirow[t]{2}{*}{ Coffee } & \multirow[t]{2}{*}{ Adulteration } & Monakhova et al. (2015) \\
\hline & & & Wei et al. (2012) \\
\hline & \multirow[t]{5}{*}{ Honey } & \multirow{3}{*}{ Adulteration } & Spiteri et al. (2015) \\
\hline & & & Donarski, Roberts, and Charlton (2010) \\
\hline & & & Spiteri et al. (2017) \\
\hline & & \multirow[t]{2}{*}{ Geographical Origin } & Donarski, Jones, and Charlton (2008) \\
\hline & & & Donarski, Jones, Harrison, Driffield, and Charlton (2010) \\
\hline & $\begin{array}{l}\text { Non-alcoholic } \\
\text { beverages }\end{array}$ & Adulteration & Ackermann et al. (2017) \\
\hline & Oils and Fats & Authenticity and botanical origin & Guyader et al. (2018) \\
\hline & \multirow[t]{4}{*}{ Olive oil } & Quality, Authenticity, Geographical Origin & Mannina and Sobolev (2011) \\
\hline & & Geographical Origin & Mannina, Marini, Gobbino, Sobolev, and Capitani (2010) \\
\hline & & Climate data & Rongai et al. (2017) \\
\hline & & Adulteration & Mannina et al. (2009) \\
\hline & Wine & $\begin{array}{l}\text { Quality, Authenticity, Geographical Origin, Grape Variety, } \\
\text { Year of vintage }\end{array}$ & Godelmann et al. (2013) \\
\hline & Vinegars & Authenticity, Vinegar types & $\begin{array}{l}\text { Boffo, Tavares, Ferreira, \& Ferreira, 2009; Consonni, } \\
\text { Cagliani, Rinaldini, \& Incerti, } 2008\end{array}$ \\
\hline & Spices & Adulteration & Petrakis, Cagliani, Polissiou, \& Consonni, 2015; Yilmaz, 2010 \\
\hline & Caviar & Authenticity & Heude et al. (2016) \\
\hline HR-MAS & Fish & Freshness & Heude, Lemasson, Elbayed, and Piotto (2015) \\
\hline \multirow[t]{3}{*}{ FoodScreener } & \multirow[t]{2}{*}{ Fruit Juice } & $\begin{array}{l}\text { Fruit and product type differentiation, Adulteration, } \\
\text { Geographical Origin, Fruit mixture }\end{array}$ & Spraul et al. (2009a) \\
\hline & & Adulteration & Spraul, Schafer, Shutz, Fang, and Link (2013) \\
\hline & Wine & Geographical origin, Grape Variety, Vintage & Spraul et al., 2009b; Monakhova et al., 2014a \\
\hline \multirow[t]{2}{*}{ Benchtop NMR } & Meat & Meat types & Jakes et al. (2015) \\
\hline & Coffee & Variety & Defernez et al. (2017) \\
\hline
\end{tabular}

the quantitation of sweeteners (sucralose or cyclamate, for instance) in non-alcoholic beverages (Ackermann et al., 2017), terpenols in coffee (Wei et al., 2012) or methylglyoxal or leptosperin in Manuka honey (Donarski, Roberts, \& Charlton, 2010; Spiteri et al., 2017).

Unfortunately, the characterization of food authenticity or adulteration cannot always be simply restricted to the identification of a single or a few marker compounds. In some cases, an analysis based on the combination of several compounds or on the entire metabolite profile of the foodstuff, representing a unique pattern characteristic of the food identity, must be used to discriminate samples according to authenticity identifying fraudulent food. In this case, the NMR based untargeted approach is used since it allows the characterization of the chemical composition of complex mixtures providing simultaneously information on a wide range of metabolites or a set of selected ones.

In recent years, NMR applications for both target and untargeted analysis of food authenticity have had a very significant contribution in the metabolomics science. Metabolomics (Fiehn, 2002) predominantly uses either spectroscopic or spectrometric techniques for a qualitative and quantitative measurement of a wide breadth of small low molecular weight metabolites in a biological sample. The use of metabolomics analysis to face complex issues relating to food fraud and food identity, including geographical origin, maturity, and variety in foodstuff, has increased significantly during the last decades.

Fig. 1 shows a typical workflow of a metabolomics study adaptable also to food quality, safety and authentication applications. Each step is fundamental and critical with important consequences for obtaining reliable and meaningful biological results.

The principles of experimental design are universal. In this phase, a number of aspects must be taken into consideration in order to maximize the possibilities of answering the research questions of interest as clearly and efficiently as possible. This includes sampling size and collection, laboratory and data analysis, identification of variables (independent, dependent and controlled) and potential biases that could be introduced. Well designed experiments together with appropriate analytical data and statistical analysis, enable researchers to increase the robustness and validity of their experimental results. The importance of these aspects on the quality of metabolomics output has been widely described in many papers and reviews (Dunn \& Ellis, 2005;
Scalbert et al., 2009; Smolinskaa, Blancheta, Buydensa, \& Wijmengaa, 2012; Wishart, 2008). Overall, each research group follows its own optimum protocol, often developed after a case-by-case evaluation of the best compromise between quality of data and throughput of analysis. In order to promote comparison of food data among different laboratories, it is recommended that any metabolomics study provides detailed information of the experimental design and the relative experimental details. Indeed, at present, the lack of standardisation in procedures is a serious drawback for inter-laboratory comparison.

The use of NMR as a methodology suitable for metabolomics is supported by its high resolution and robustness. Further considerations must be given on the source of variability when NMR-based metabolomics is used towards food authentication. Often in metabolomics projects all samples are analysed simultaneously and no or minimal consideration is needed for factors that influence acquired data over time. These factors include instrumental drift, extraction solvents and NMR acquisition buffers. Instrumental drift (minor diminishing of the magnetic field strength of an NMR instrument), except in the most extreme of circumstances, will not impact comparability of data over time. Using a field strength of $400 \mathrm{MHz}$ as a example, a modern instrument can expect to have a magnetic field drift of less than $4 \mathrm{~Hz}$ per hour, as reported by technical specifications of instruments producers, corresponding to no greater than $35000 \mathrm{~Hz}$ per annum. This impact equates to a change in field strength of less than $0.01 \%$, and will not be observed when comparing historical and newly acquired data. Instrumental drift that occurs during experimental acquisition is typically controlled using a lock routine, where the drift observed in a secondary nucleus, often deuterium, is used to correct that of the observed nucleus. Furthermore, it is typical to bin NMR spectra to defined regions based on chemical shift. The universal nature of the chemical shift dictates that changes in magnetic field strength will not influence data. Extraction solvents and NMR acquisition buffers are considered together. These factors are controlled through the use of good laboratory practise, using precise and accurate volumes and masses. It is also recommended that where possible large batches of NMR acquisition buffers are prepared, and aliquots stored in suitable conditions (e.g. $-20^{\circ} \mathrm{C}$ ). The incorporation of a known, previously analysed sample in all batches and checks to assure that these data are still comparable, is 


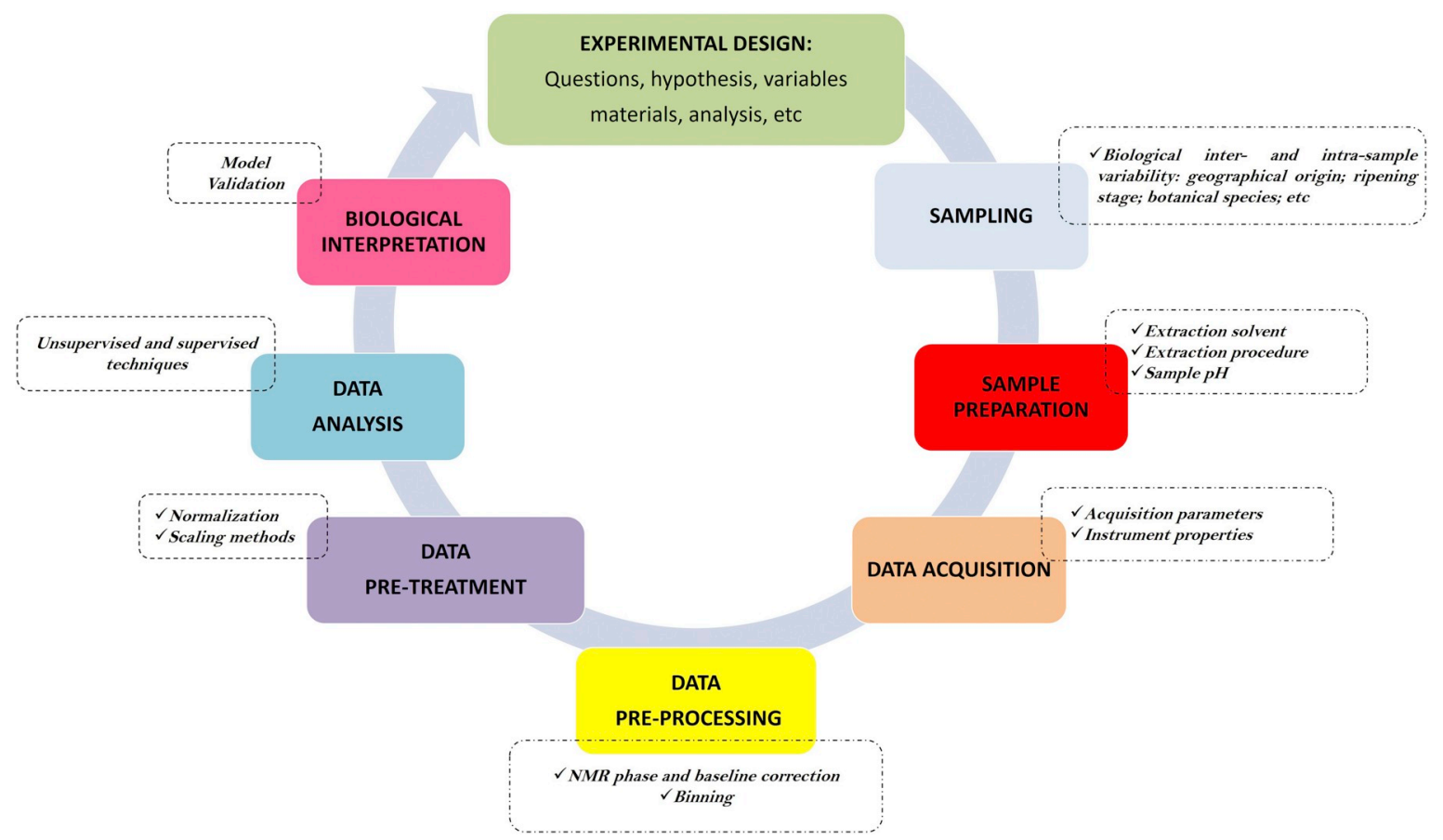

Fig. 1. Typical metabolomics workflow and sources of variability (dashed box) in food analysis. Sources for data pre-processing and pre-treatment phases refer specifically to a NMR-based study.

the recommended practice.

The metabolomics workflow ends with the multivariate statistical analysis of the quantitative data and the subsequent biochemical interpretation of the experimental results and thus the answer to the study questions (van der Greef \& Smilde, 2005; Wishart, 2008). Generally, explorative unsupervised techniques, such as Principal Component Analysis (PCA), are applied to evidence outliers or natural grouping of samples, while supervised multivariate techniques such as Partial Least Squares Discriminant Analysis (PLS-DA) are required to create classification or prediction models. Since different statistical methods use specific data treatment and a set of unique assumptions, it is recommended to verify metabolic difference by using a variety of tests.

It is therefore evident that the usefulness of metabolomics in obtaining reliable and biological meaningful results for food authentication largely depends on multiple factors and overall on the ability to set up appropriate quality control strategies to monitor the robustness of the metabolomics workflow, thus minimizing the impact of error sources on the data interpretation.

A prominent example of the unique information available from the application of NMR and metabolomics regards honey. Verifying the geographical origin of honey samples from the French island of Corsica by applying a ${ }^{1} \mathrm{H}$ NMR statistical protocol has been demonstrated in a study performed on 118 samples collected from a range of European countries and regions (Donarski et al., 2008). The subsequent statistical model, built using a combination of PLS-DA and genetic algorithm, was able to correctly identify honey samples as originating from Corsica (overall, correct classification rates, from a cross validated model, were $96.2 \%)$. A follow-up study which combined data from a second year of study investigated the variables used in a new classification algorithm and identified several biomarkers that were present in specific honey floral types (e.g. kynurenic acid was identified by a genetic algorithm as an indirect marker of geographical origin) (Donarski, Jones, Harrison, Driffield, \& Charlton, 2010). Spiteri et al. (2015) reported a bulk screening ${ }^{1} \mathrm{H}$ NMR study of 205 commercial honey samples against 518 reference honeys by using Independent Components Analysis. As a result, a significant number of the commercial samples were found to fall outside the classification model created with authentic samples, suggesting a possible addition of exogenous sugars.

NMR spectroscopy has proven to be an extraordinary tool also for the characterization of olive oils in terms of quality, authenticity and geographical origin (Mannina \& Sobolev, 2011). A geographical classification of Mediterranean virgin olive oils produced in three consecutive years was performed on 896 authentic samples by ${ }^{1} \mathrm{H}$ NMR spectroscopy and different statistical models (Mannina et al., 2010). The potential influence of the historical meteorological parameter averages was investigated by the ${ }^{1} \mathrm{H}$ NMR-metabolomics approach on more than 200 samples of extravirgin olive oils collected over a fouryear period in Italy, European and non-European countries. Besides suggesting the possible use of this approach for olive oils geographical origin prediction, the results of this study indicated also a possible application to the assessment of correlation between the profile of olive oils and climate data (Rongai et al., 2017). Blending of virgin olive oil with low-quality hazelnut oil can be unmasked by the statistical elaboration of ${ }^{1} \mathrm{H}$ NMR signals from fatty acid chains, squalene, and $\beta$ sitosterol (Mannina et al., 2009). Finally, it is worth reminding that, since NMR does not require prior knowledge about the compounds present in the sample, it is ideally suited to detect unexpected changes in the metabolite profile of food, such as the addition of new adulterants, without the need of an a priori hypothesis.

${ }^{1} \mathrm{H}$ NMR spectroscopy in combination with multivariate data analysis can be successfully used also to achieve information on various aspect of wine quality such as the authenticity, grape variety, geographical origin, and the year of vintage (Godelmann et al., 2013).

\section{Routine cost-effective NMR approach}

\subsection{NMR validation and quality assurance}

NMR method is becoming more and more popular within the food testing community, proving to be also a well suited technique for quality control (QC) applications, both as targeted or non-targeted approach. Indeed, a single NMR sample measurement can answer many authenticity issues that could not be resolved by conventional methods. 
The high throughput of NMR spectroscopy enables the analyst to quickly measure the large number of samples required to populate a comprehensive database. The whole spectrum is measurable with a high reproducibility, even between laboratories (Thomas, 2016). This is possible thanks to precise standard operating procedures for sample preparation and new technological developments that have minimised variability between laboratories. These include: precise monitoring of the sample temperature; automation of settings such as tuning and matching of the probe; gradient shimming to ensure magnetic field homogeneity. The whole spectrum can be considered as a reproducible fingerprint, without sophisticated pre-processing before statistical evaluation. To finalise the recognition of non-targeted NMR methods, standardisation is the final goal. Normalisation bodies are currently considering the potential of these methods including the wider requirements relating to data accessibility and database maintenance (Thomas, 2016).

Therefore, the potential of NMR is not only to measure the major signals from the main metabolites but also to do so without the influence of spectral artefacts and inconsistencies such as electrical noise, peak width differences and chemical shift modification. Spectra can then be compared to each other, and by comparing an unknown spectrum with a series of reference spectra from a well-defined population, defaults are detected, without any a priori selection of variables. Such models of classification or verification are not performed as "black-box" since the user can move back to the spectral fingerprint and identify which signal is responsible for the deviation.

Through the integration of the NMR methodology inside a Quality Management System and with the use of periodic QC, the laboratory can even obtain Third Party accreditation for its NMR techniques (Thomas, 2016). On the technical level, it should periodically evaluate its performance of tests. The performance validation of the analytical method must encompass all stages of preparation, extraction and analysis encountered by samples during routine analyses. Performance characteristics include: selectivity, measuring range, linearity, sensitivity, limit of detection, limit of quantitation, robustness, precision, and accuracy as described in EUROLAB Technical Report 1/2015 (Schönberger et al., 2015). Additionally, laboratories should start participating in collaborative trials and making use of various International Proficiency Testing Schemes (PTS) for monitoring their analytical performance and compares the results to laboratories worldwide. They include the Pro-PTS organised by Eurofins laboratory (Thomas, 2018) and the Food Analysis Performance Assessment Scheme (FAPAS) from Fera.

\subsection{Current routine applications}

A considerable number of literature reports have highlighted the use of NMR methodology for the study of food samples and its development of routine use in this field. This way of working is now adopted in different food control laboratories where NMR is in the portfolio of routine methods for control of products to protect producers and consumers. Applications include, for example, the control of honey (detection of added sugar, geographical and botanical origin confirmation), beverages (Cuny et al., 2008; Lamanna, Braca, Di Paolo, \& Imparato, 2011; Li et al., 2016; Spraul et al., 2009a, 2009b), coffee, fats and oils (through the use of ${ }^{13} \mathrm{C}$ NMR it is possible to observe the alpha or beta positional distribution of fatty acid chains in the glycerol moiety (Guyader et al., 2018; Mannina \& Sobolev, 2011), vinegars (Boffo et al., 2009; Consonni et al., 2008), spices (detection of added forbidden dyes or detection of other spices and herbs or natural adulterants (Petrakis et al., 2015; Yilmaz, 2010)) and many other applications (detection of non-authorised processes for dried fruits such as forbidden/non-declared cleaning or drying agents - unpublished studies from Eurofins). Further details on how NMR could be use in food QC are provided in the following sections.

A NMR-based platform that uses targeted and untargeted analysis called FoodScreener ${ }^{\mathrm{rm}}$, has been developed. It allows the simultaneous evaluation of different quality and authenticity related-parameters. To date, three different modules of the FoodScreener ${ }^{\mathrm{TM}}$ platform are available, namely: Spin Generated Fingerprint-Profiling (SGFProfiling $^{\mathrm{TM}}$ ) for fruit juice and Wine-Profiling ${ }^{\mathrm{TM}}$ for wine. As demonstration of its commercial application and its increasing acceptance as routine use, it can be reported the recent decision of the Hungarian Ministry of Agriculture (August 2017) to start a program in order to authenticate and identify Hungarian wines using NMR FoodScreenerWine-Profiling ${ }^{\mathrm{TM}}$. The cost of single analysis can vary in relation to the possibility to buy and use the platform or to send the sample to a laboratory equipped with FoodScreener. The cost of a complete single analysis is about $200-250 €$ according to the food matrix.

The SGF-Profiling ${ }^{\mathrm{TM}}$ module represents a heterogeneous collection of statistical models which can be applied consecutively to one single spectrum (Spraul et al., 2009a). Depending on the model used, the following are predictable: fruit and product type differentiation, adulteration by sugar or acid addition, geographical origin and fruit mixture. Furthermore, information on the quantification of substances can be carried out. In particular, targeted analysis provides information on the content of sugars (glucose, fructose and sucrose), the total acidity of the juice (citric, malic, isocitric and quinic acid), perishable indicators (ethanol, fumaric acid, lactate) and process control parameters (galacturonic acid, phlorin). The values are compared to reference standards. The screening is based on a database composed by thousands of NMR spectra from authentic juices. Finally, the combination of SGFProfiling $^{\mathrm{TM}}$ and the associated software JuiceScreener ${ }^{\mathrm{TM}}$ provides the analytical report in which the analytical answer and results are summarized. Regarding the untargeted analysis, both the assessment of concentration deviation of hundreds of compounds and unexpected ingredients are detectable to verify adulteration and unknown fraud (Spraul et al., 2013). The application of SGF- Profiling ${ }^{\mathrm{TM}}$ is well established for the routine use analysis of fruit products (juices, concentrates, nectars, purees ...).

This technology has been extended to other types of food material such as wine (Monakhova et al., 2014a; Spraul et al., 2009b). WineProfiling $^{\mathrm{TM}}$ is a method for wine analysis. The comparison of the spectroscopic fingerprint obtained for each individual sample with that of a large database of authentic wine samples allows to answer question on the composition, geographical origin, grape variety and vintage. Although this procedure had been already developed for fruit juice analysis, it was further optimized for wine and alcoholic beverages in general. In particular, to overcome the need to eliminate the major signals (water and ethanol), a methodology was developed which can suppress both signals from water and ethanol during NMR experiment without losing signals outside those regions (Minoja \& Napoli, 2014). In a similar way to SGF-Profiling ${ }^{\mathrm{TM}}$, Wine-Profiling ${ }^{\mathrm{TM}}$ provides both targeted and untargeted analysis. The former is performed through the quantification of 56 parameters per sample and their comparison with official reference values, while the latter is carried out through verification models able to detect any deviation from authentic reference data.

New NMR benchtop instrumentations, low field but high resolution, represent a new frontier in the food authentication field. Several manufacturers currently offer benchtop instruments that operate at a proton frequency between 45 and $90 \mathrm{MHz}$. These instruments have significantly lower purchase and maintenance costs than conventional NMR instruments, do not require liquid nitrogen and helium cryogens and do not require specialised staff for their operation. Although some NMR phenomenon are adversely effected at lower field strengths, e.g. sensitivity is lower and spectral overlap is greater, these instruments are still incredibly powerful for food authentication analysis.

The utilisation of low field instrumentation for food analysis was presented at the 7th International Symposium on Recent Advances in Food Analysis (RAFA 2015). The example of rapid analysis for spirit authentication was shown. Under Council Regulation (EEC) $\mathrm{N}^{\circ} .110 /$ 
2008 of the European Parliament and of the Council of 15 January 2008 "laying down general rules on the definition, description and presentation of spirit drinks", minimum alcoholic strengths for spirit drinks are set. The alcohol content of a spirit drink can be altered by two common fraud types: extension (diluting a spirit drink with water) and counterfeiting (replacing a genuine product with a copy). Therefore, the ability to accurately assess the alcoholic strength of a spirit drink can assist in detection of these frauds. The quantitative nature of the NMR resonances can be theoretically exploited to determine the alcoholic strength of a sample without the requirement for an internal standard. In practise, the assumptions made are non-trivial and a correction coefficient must be calculated to routinely use ${ }^{1} \mathrm{H}$ NMR spectroscopy for alcohol content determination. A study (unpublished) using an early generation low field spectrometer could accurately calculate ethanol content within 1.96\% (CI 95\%), whereas results from a high field $500 \mathrm{MHz}$ NMR spectrometer were approximately 20 -fold more accurate. It is expected that a significant increase on accuracy will be observed as further advances are made in instrumentation.

Benchtop NMR provides also a fast, direct, and user-friendly method to determine the fat and oil content in foodstuffs. The technique is based on measurement of the NMR response obtained from oil (fat) in the product, and quantification of its content by calibration. The study performed by Jakes et al. (2015) is a good demonstration on the ability of low field NMR spectroscopy to differentiate beef from horse meat. The lipid constituents of beef and horse samples analysed by low-field NMR were sufficiently different to enable the creation of classification models. When these models were challenged with blind samples (ones that had not been used in model creation) excellent classification results were obtained. Another successful example of food analysis by benchtop NMR is provided by Defernez et al. (2017). In this study, a methodology for distinguishing Arabica and Robusta coffee by using high field NMR spectroscopy (Monakhova et al., 2015) was transferred to a $60 \mathrm{MHz}$ low field spectrometer. Arabica coffee beans command a higher price than Robusta beans, therefore an incentive exists for unscrupulous traders to substitute Robusta beans for Arabica. Although it is possible to differentiate whole Arabica and Robusta beans by eye due to morphological differences, this is not possible on roasted and ground coffee. Chemically, the beans can be distinguished through detection of the compound 16-O-methylcafestol (16-OMC), which is found exclusively in Robusta beans. Therefore, authentic samples of Arabica do not contain 16-OMC and this compound can be used to detect adulteration/substitution with Robusta. The developed low-field NMR methodology was rapid, with a total time for analysis, including extraction and data acquisition, of approximately $45 \mathrm{~min}$. It could detect adulteration of Arabica with Robusta when the adulteration level was greater than $10-20 \%$. The methodology was used to perform a small market survey, the results of which showed no adulteration.

\subsection{Problematic matrices for NMR}

Liquid foods, such as beverages, vegetable oils and fully soluble foodstuffs like honey are very simple to analyse by ${ }^{1} \mathrm{H}$ NMR because they do not require specific preparation. Therefore, they can be analysed with no to minimal pre-treatment and high resolution spectra are usually obtained. Some experimental difficulties due to the presence of the signals of the most abundant components (water in fruit juices, ethanol and water in wine and beer, etc.) have been resolved using specific pulse sequences to suppress these signals (Mannina et al., 2016).

Non-homogeneous liquid foods usually do not show sufficiently resolved NMR spectra, and additional extraction procedures may be necessary to extract and study their components. Due to its nature, milk can be considered one of the most complicated foods to study. Milk is an emulsified colloid consisting of small globules of fat and protein suspended in water, the principal component of milk. Therefore, a wellresolved ${ }^{1} \mathrm{H}$ NMR spectrum is difficult to obtain. In literature, only one example of ${ }^{1} \mathrm{H}$ NMR analysis of milk without any pretreatment is reported (Hu, Furihata, Ito-Ishida, Kaminogawa, \& Tanokura, 2004). Other studies of milk have required sample pretreatment such as the use of spin filters with specific cut-off to remove residual lipids and protein (Sundekilde, Poulsen, Larsen, \& Bertram, 2013), the extraction of triacylglycerols, and/or adjustment of the $\mathrm{pH}$ before the acquisition of ${ }^{13} \mathrm{C}$ and ${ }^{31} \mathrm{P}$ NMR spectra (Andreotti, Lamanna, Trivellone, \& Motta, 2002; Belton \& Lyster, 2009). The "traditional" frauds encountered in milk include: watering, lactoserum addition, species blend, old milk reuse, etc. More recently, other issues have involved the addition of nitrogen containing compounds (melamine, dicyandiamide, protein hydrolysates, etc.) to artificially increase the protein content (as determined from total nitrogen). The current approach offered by routine laboratories to control the authenticity of milk and milk powder consist of doing 2 extractions in parallel in order to cover all potential adulterations (unpublished):

- one extraction in water to monitor the sugar profile (with the possibility of identifying delactosed milk and to observe if sucrose or maltodextrin have been added), to detect protein hydrolysate and to control microbial degradation,

- one extraction in dimethyl sulfoxide to monitor the fat content (whole, semi-skimmed, skimmed), to detect addition of foreign fats, to identify the thermal treatment (pasteurized or sterilised) or to detect melamine or other $\mathrm{N}$-enhancers.

Solid food samples require specific preparation, including sampling, conservation and homogenization for extraction, to be analysed by liquid state ${ }^{1} \mathrm{H}$ NMR. The extraction procedure is a critical step contributing significantly to the final quali-quantitative profile composition revealed by NMR. As an illustration of this extraction procedure, untargeted screening methods have been developed to authenticate caviar (Heude et al., 2016) as a tool to protect the PGI (Protected Geographical Indication) "caviar d'Aquitaine". Using an untargeted approach, authentic caviar d'Aquitaine and foreign origin caviar were analysed using ${ }^{1} \mathrm{H}$ NMR spectroscopy. This method was validated and implemented in control laboratory.

Another problem source for NMR is the widespread presence of paramagnetic metals (i.e. $\mathrm{Fe}^{3+}, \mathrm{Mn}^{2+}$ ) in various foodstuffs, namely spices, cereals, dried fruits and meat. During the extraction procedure, these cations can be co-extracted and give rise to the broadening of ${ }^{1} \mathrm{H}$ signal of metabolites, leading to decreased resolution.

The direct application of the High Resolution Magic Angle Spinning (HR-MAS) for products without any pretreatment is possible for semisolid foodstuff (Ritota, Marini, Sequi, \& Valentini, 2010). Such technology has been used to control fish freshness (Heude et al., 2015) but because of the lack of automation, currently applications are limited to specific studies.

\section{Future perspective}

Taking into account the observations reported so far, we think two ways in which NMR will be better implemented into routine use, i.e. by the development of further FoodScreener ${ }^{\mathrm{TM}}$ type platforms for untargeted applications and the development of targeted approaches for low-field benchtop instruments.

The development of FoodScreener ${ }^{\mathrm{TM}}$ platforms will rely on the setting up of NMR food databases, widely shared experimental protocols and will benefit from the interface with other analytical platforms. In order to create a comprehensive database of NMR foodstuffs, it is important to report the NMR profile of the entire foodstuff with the relative assignment. In this way, the matrix effect and relative contribution of different components will be taken into account. The existing NMR databases regarding foodstuffs are focused on single components or classes of compounds with rich information obtained from the analysis of standard compounds. Only NMR data on single isolated 
components are available and not the entire NMR profiling. Considering the matrix effect on NMR spectral parameters such as chemical shifts and linewidth, the spectrum of an isolated compound may not coincide with the spectrum of the same component in a food matrix. Thus, addition of the standard in the sample (commonly referred to as over-spiking) is a one of the most robust methods to verify the presence of food components. Another important aspect that worth including in the database is an exhaustive description of the food sample in terms of its natural variability, processing, storage, and all other factors responsible for food identity and, therefore, for its NMR spectrum.

An example of existing food databases is FooDB (FooDB, http:// foodb.ca) that aggregates data from different sources reporting food constituents and their chemical and biological properties, mostly for unprocessed foodstuffs. Unfortunately, the general classification of food types does not include some essential aspects relative to variety, processing, etc that determine the food identity. For instance, tea (Camellia sinensis) is reported as a single type product without taking into consideration different fermentation types and varieties.

An attempt to create a comprehensive database including food NMR spectra and data on their origin, composition, variety etc. is being undertaken by the Italian Group of Magnetic Resonance in Food Science (e-ALIERB OpenLab, 2016 http://www.e-alierb.it/). Outputs and recommendations will be drawn from this research to provide implementation guidelines and it is recommended that these conclusions are published in a peer reviewed journal.

The creation of comprehensive NMR databases of foodstuffs is hindered by the fragmentation of experimental approaches used until to now. Different extraction procedures, solvents, quantification and statistical analysis have been applied for the same foodstuff that results in non-comparable NMR profiles. Therefore, an international collaboration between researchers is necessary to unify different approaches and can be a key element in the development of a food NMR database that is constantly, or at least periodically, updated.

The combination of data from different platforms (NMR and other analytical methods) is a powerful strategy to enrich the final information content regarding food identity and thus improve the identification of food frauds followed by routine applications. The benefit of combining two untargeted methods has been recently illustrated on honey by Spiteri et al. (2016). Using data from two analytical platforms, namely NMR and high resolution mass spectrometry (HRMS), and two separate HRMS technologies, honey samples were analysed with the aim of determining their botanical origins. No one of the PCA models built with individual NMR or MS data set was able to discriminate samples in terms of botanical origin. Then, mid-level fusion of the data was explored in two ways to improve the discrimination: fusion of PCA scores from the combined data sets or after selection of variables by PLS-DA. In both cases, it was possible to improve the discrimination so that botanical origin could be assigned without the need for pollen analysis.

Another example of the benefit of fusing NMR data with alternative techniques has been provided by Monakhova et al. (2014b). The authors evaluated the combination of discrete isotopic data with untargeted NMR spectrum to have better control of wines. Both techniques are known to provide useful information to the characterization of wine: ${ }^{1} \mathrm{H}$ NMR spectroscopy can be used to build robust classification models for grape variety, year of vintage and geographical origin, while stable isotope ratio analysis is a good source of chemical information for the authenticity assessment of food products. By combining these two methodologies, improvement of classification rates of wine was achieved: $100 \%$ for the determination of geographical origin $(60-70 \%$ correct prediction was obtained with stable isotope data alone and 82-89\% with ${ }^{1} \mathrm{H}$ NMR spectroscopy) and $99 \%$ for the vintage of wine (from 88 to $97 \%$ with ${ }^{1} \mathrm{H}$ NMR).

Benchtop NMR instrumentation has the potential to be a breakthrough technology in the field of food authentication within the industry. It utilises the power of NMR spectroscopy but with significantly reduced infrastructure requirements and costs. It can be applied in either the targeted or the untargeted approach. In the targeted approach, it is possible to routinely quantify components within complex mixtures and to translate academic research into deployable solutions. In the untargeted approach, models of normality can be rapidly developed and used by industry to confirm their products are consistent with previous batches. This application of spectroscopic technology is not new, and has been implemented using profiling technologies such as near and Fourier Transform infra-red (NIR, FT-IR) spectroscopy. The unique advantage of routine profiling using low-field NMR spectroscopy though, is the interpretability of signals to specific chemicals. This is because NMR phenomenon is directly transferable from benchtop instrumentation to high field, laboratory based instrumentation and the advantages they provide. For example, in a theoretical case where a food sample is shown to be inconsistent by benchtop NMR, the same sample analysed on a high field instrument would show the same inconsistency, enabling rapid unknown identification. Once identified, intelligence can be applied to the inconsistent sample to determine what action, if any is required. Studies that apply both low of high field analysis are recommended to demonstrate the utility of this approach.

\section{Acknowledgements}

The research leading to these results has received funding from the European Union's Seventh Framework Programme for research, technological development and demonstration under grant agreement No. 613688.

It was also realized with funds received from the following agencies: Italian Ministry of Education, Universities and Research - Dipartimenti di Eccellenza - L. 232/2016.

\section{References}

Ackermann, S. M., Dolsophon, K., Monakhova, Y. B, Kuballa, T, Reusch, H., Thongpanchang, T., et al. (2017). Automated multicomponent analysis of soft drinks using $1 \mathrm{D}{ }^{1} \mathrm{H}$ and $2 \mathrm{D}{ }^{1} \mathrm{H}-{ }^{1} \mathrm{H} J$-resolved NMR spectroscopy. Food Analytical Methods, 3, 827-836.

Andreotti, G., Lamanna, R., Trivellone, E., \& Motta, A. (2002). ${ }^{13} \mathrm{C}$ NMR spectra of TAG: An easy way to distinguish milks from different animal species. Journal of the American Oil Chemists' Society, 79, 123-127.

Belton, P. S., \& Lyster, R. L. J. (2009). ${ }^{31}$ P nuclear magnetic resonance spectra of milk from various species. Journal of Dairy Research, 58, 443-451.

Boffo, E. F., Tavares, L. A., Ferreira, M. M. C., \& Ferreira, A. G. (2009). Classification of Brazilian vinegars according to their ${ }^{1} \mathrm{H}$ NMR spectra by pattern recognition analysis. LWT - Food Science and Technology, 42, 1455-1460.

Consonni, R., Cagliani, L. R., Rinaldini, S., \& Incerti, A. (2008). Analytical method for authentication of traditional balsamic vinegar of Modena. Talanta, 75, 765-769.

Cuny, M., Vigneau, E., Le Gall, G., Colquhoun, I., Lees, M., \& Rutledge, D. N. (2008). Fruit juice authentication by ${ }^{1} \mathrm{H}$ NMR spectroscopy in combination with different chemometrics tools. Analytical and Bioanalytical Chemistry, 390, 419-427.

Defernez, M., Wren, E., Watson, A. D., Gunning, Y., Colquhoun, I. J., Le Gall, G., et al (2017). Low-field ${ }^{1}$ H NMR spectroscopy for distinguishing between Arabica and Robusta ground roast coffees. Food Chemistry, 216, 106-113.

Donarski, J. A., Jones, S. A., \& Charlton, A. J. (2008). Application of cryoprobe ${ }^{1} \mathrm{H}$ nuclear magnetic resonance spectroscopy and multivariate analysis for the verification of Corsican honey. Journal of Agricultural and Food Chemistry, 56, 5451-5456.

Donarski, J. A., Jones, S. A., Harrison, M., Driffield, M., \& Charlton, A. J. (2010a) Identification of botanical biomarkers found in Corsican honey. Food Chemistry, 118 987-994.

Donarski, J. A., Roberts, D. P. T., \& Charlton, A. J. (2010b). Quantitative NMR spectroscopy for the rapid measurement of methylglyoxal in manuka honey. Analytical Methods, 10, 1479-1483.

Dunn, W. B., \& Ellis, D. I. (2005). Metabolomics: Current analytical platforms and methodologies. Trends in Analytical Chemistry, 24, 285-294.

Fiehn, O. (2002). Metabolomics - the link between genotypes and phenotypes. Plant Molecular Biology, 48, 155-171.

FooDB (2017). http://foodb.ca/, Accessed date: 26 July 2019.

Godelmann, R., Fang, F., Humpfer, E., Schutz, B., Bansbach, M., Schafer, H., et al. (2013) Targeted and nontargeted wine analysis by ${ }^{1} \mathrm{H}$ NMR spectroscopy combined with multivariate statistical analysis. Differentiation of important parameters: Grape variety, geographical origin, year of vintage. Journal of Agricultural and Food Chemistry, 61, 5610-5619.

van der Greef, J., \& Smilde, A. K. (2005). Symbiosis of chemometrics and metabolomics: Past, present, and future. Journal of Chemometrics, 19, 376-386. 
Guyader, S., Thomas, F., Portaluri, V., Jamin, E., Akoka, S., Silvestre, V., et al. (2018). Authentication of edible fats and oils by non-targeted ${ }^{13} \mathrm{C}$ INEPT NMR spectroscopy. Food Control, 91, 216-224.

Heude, C., Elbayed, K., Jezequel, T., Fanuel, M., Lugan, R., Heintz, D., et al. (2016). Metabolic characterization of caviar specimens by ${ }^{1} \mathrm{H}$ NMR spectroscopy: Towards caviar authenticity and integrity. Food Analytical Methods, 9, 3428-3438.

Heude, C., Lemasson, E., Elbayed, K., \& Piotto, M. (2015). Rapid assessment of fish freshness and quality by ${ }^{1}$ H HR-MAS NMR spectroscopy. Food Analytical Methods, 8, 907-915.

Hu, F., Furihata, K., Ito-Ishida, M., Kaminogawa, S., \& Tanokura, M. (2004). Nondestructive observation of bovine milk by NMR spectroscopy: Analysis of existing states of compounds and detection of new compounds. Journal of Agricultural and Food Chemistry, 52, 4969-4974.

Jakes, W., Gerdova, A., Defernez, M., Watson, A. D., McCallum, C., Limer, E., et al. (2015). Authentication of beef versus horse meat using $60 \mathrm{MHz}{ }^{1} \mathrm{H}$ NMR spectroscopy. Food Chemistry, 175, 1-9.

Lamanna, R., Braca, A., Di Paolo, E., \& Imparato, G. (2011). Identification of milk mixtures by ${ }^{1} \mathrm{H}$ NMR profiling. Magnetic Resonance in Chemistry, 49, S22-S26.

Li, Q., Yu, Z., Zhu, D., Meng, X., Pang, X., Liu, Y., et al. (2016). The application of NMRbased milk metabolite analysis in milk authenticity identification. Journal of the Science of Food and Agriculture, 97, 2875-2882.

Mannina, L., D'Imperio, M., Capitani, D., Rezzi, S., Guillou, C., Mavromoustakos, T., et al. (2009). ${ }^{1} \mathrm{H}$ NMR-based protocol for the detection of adulterations of refined olive oil with refined hazelnut oil. Journal of Agricultural and Food Chemistry, 57, 11550-11556.

Mannina, L., Marini, F., Antiochia, R., Cesa, S., Magrì, A., Capitani, D., et al. (2016). Tracing the origin of beer samples by NMR and chemometrics: Trappist beers as a case study. Electrophoresis, 37, 2710-2719.

Mannina, L., Marini, F., Gobbino, M., Sobolev, A. P., \& Capitani, D. (2010). NMR and chemometrics in tracing European olive oils: The case study of Ligurian samples. Talanta, 80, 2141-2148.

Mannina, L., \& Sobolev, A. P. (2011). High resolution NMR characterization of olive oils in terms of quality, authenticity and geographical origin. Magnetic Resonance in Chemistry, 49, S3-S11.

Mannina, L., Sobolev, A. P., Aru, V., Bellomaria, A., Bertocchi, F., Botta, B., et al. (2017). Methodologies in food analysis. In M. Locatelli, \& C. Celia (Eds.). Analytical chemistry: Developments, applications and challenges in food science (pp. 103-156). New York: Nova Science Publishers, Inc.

Minoja, A. P., \& Napoli, C. (2014). NMR screening in the quality control and nutraceuticals. Food Research International, 63, 126-131.

Monakhova, Y. B., Godelmann, R., Hermann, A., Kuballa, T., Cannet, C., Schäfer, H., et al. (2014b). Synergistic effect of the simultaneous chemometric analysis of ${ }^{1} \mathrm{H}$ NMR spectroscopic and stable isotope (SNIF-NMR, ${ }^{18} \mathrm{O},{ }^{13} \mathrm{C}$ ) data: Application to wine analysis. Analytica Chimica Acta, 833, 29-39.

Monakhova, Y. B., Ruge, W., Kuballa, T., Ilse, M., Winkelmann, O., Diehl, B., et al. (2015). Rapid approach to identify the presence of Arabica and Robusta species in coffee using ${ }^{1}$ H NMR spectroscopy. Food Chemistry, 182, 178-184.

Monakhova, Y. B., Schutz, B., Schafer, H., Spraul, M., Kuballa, T., Hahan, H., et al. (2014a). Validation studies for multicomponent quantitative NMR analysis: The example of apple fruit juice. Accreditation and Quality Assurance, 19, 17-29.

Petrakis, E. A., Cagliani, L. R., Polissiou, M. G., \& Consonni, R. (2015). Evaluation of saffron (Crocus sativus L.) adulteration with plant adulterants by ${ }^{1} \mathrm{H}$ NMR metabolite fingerprinting. Food Chemistry, 173, 890-896.

Proietti, N., Capitani, D., Aru, V., Bellomaria, A., Bertocchi, F., Botta, B., et al. (2017). NMR Applications in food analysis - Part B. In M. Locatelli, \& C. Celia (Eds.). Analytical chemistry: Developments, applications and challenges in food science (pp. 255296). New York: Nova Science Publishers, Inc.

Ritota, M., Marini, F., Sequi, P., \& Valentini, M. (2010). Metabolomic characterization of Italian sweet pepper (Capsicum annum L.) by means of HRMAS-NMR spectroscopy.
Journal of Agricultural and Food Chemistry, 58, 9675-9684.

Rongai, D., Sabatini, N., Del Coco, L., Perri, E., Del Re, P., Simone, N., et al. (2017). ${ }^{1} \mathrm{H}$ NMR and multivariate analysis for geographic characterization of commercial extra virgin olive oil: A possible correlation with climate data. Foods, 6, 96.

Scalbert, A., Brennan, L., Fiehn, O., Hankemeier, T., Kristal, B. S., van Ommen, B., et al (2009). Mass-spectrometry-based metabolomics: Limitations and recommendations for future progress with particular focus on nutrition research. Metabolomics, 5, 435-458.

Schönberger, T., Monakhova, Y. B., Lachenmeier, D. W., Walch, S., \& Kuballa, T. NonProfit Expert Team (NEXT) -NMR working group Germany. (2015). Guide to NMR method development and validation-Part II: Multivariate data analysis. Brussels, Belgium: EUROLAB General Secretariat.

Smolinskaa, A., Blancheta, L., Buydensa, L. M. C., \& Wijmengaa, S. S. (2012). NMR and pattern recognition methods in metabolomics: From data acquisition to biomarker discovery: A review. Analytica Chimica Acta, 750, 82-97.

Sobolev, A. P., Circi, S., \& Mannina, L. (2016). Advances in nuclear magnetic resonance spectroscopy for food authenticity testing. In G. Downey (Ed.). Advances in food authenticity testing (pp. 147-170). United Kingdom: Woodhead Publishing Elsevier Ltd.

Sobolev, A. P., Mannina, L., Aru, V., Bellomaria, A., Bertocchi, F., Botta, B., et al. (2017). NMR applications in food analysis - Part A. In M. Locatelli, \& C. Celia (Eds.). Analytical chemistry: Developments, applications and challenges in food science (pp. 157254). New York: Nova Science Publishers, Inc.

Spiteri, M., Dubin, E., Cotton, J., Poirel, M., Corman, B., Jamin, E., et al. (2016). Data fusion between high resolution ${ }^{1} \mathrm{H}-\mathrm{NMR}$ and mass spectrometry: A synergetic approach to honey botanical origin characterization. Analytical and Bioanalytical Chemistry, 408, 4389-4401.

Spiteri, M., Jamin, E., Thomas, F., Rebours, A., Lees, M., Rogers, K. M., et al. (2015). Fast and global authenticity screening of honey using ${ }^{1} \mathrm{H}-\mathrm{NMR}$ profiling. Food Chemistry, 189, 60-66.

Spiteri, M., Rogers, K. M., Jamin, E., Thomas, F., Guyader, S., Lees, M., et al. (2017). Combination of ${ }^{1} \mathrm{H}$ NMR and chemometrics to discriminate Manuka honey from other floral honey types from Oceania. Food Chemistry, 217, 766-772.

Spraul, M., Schafer, H., Shutz, B., Fang, F., \& Link, M. (2013). Novel NMR-technology to assess food quality and safety. International Journal of Biological, Biomolecular, Agricultural, Food and Biotechnological Engineering, 7, 960-963.

Spraul, M., Schuz, B., Rinke, P., Koswig, S., Humpfer, E., Schafer, H., et al. (2009b). NMR based multi parametric quality control of fruit juices: SGF profiling. Nutrients, 1 148-155.

Spraul, M., Shutz, B., Humpfer, E., Mortter, M., Schafer, H., Koswig, S., et al. (2009a). Mixture analysis by NMR as applied to fruit juice quality control. Magnetic Resonance in Chemistry, 47, S130-S137.

Sundekilde, U. K., Poulsen, N. A., Larsen, L. B., \& Bertram, H. C. (2013). Nuclear magnetic resonance metabonomics reveals strong association between milk metabolites and somatic cell count in bovine milk. Journal of Diary Science, 96, 290-299.

The 7th International Symposium on Recent Advances in Food Analysishttp://www.rafa2015. eu/, Accessed date: 26 July 2019.

Thomas, F. (2016). Challenges in nuclear magnetic resonance spectroscopy based nontargeted analysis. International Symposium on standardisation of non-targeted methods for food authentication (Berlin, Germany).

Thomas, F. (2018). Pro-pts (NMR profiling technique - proficiency testing Scheme). (unpublished document, available from: the authors).

Wei, F., Furihata, K., Koda, M., Hu, F., Miyakawa, T., \& Tanokura, M. (2012). Roasting process of coffee beans as studied by nuclear magnetic resonance: Time course of changes in composition. Journal of Agricultural and Food Chemistry, 60, 1005-1012.

Wishart, D. S. (2008). Quantitative metabolomics using NMR. Trends in Analytical Chemistry, 27, 228-237.

Yilmaz, A. (2010). ${ }^{1} \mathrm{H}$ NMR metabolic fingerprinting of saffron extracts. Metabolomics, 6 , 511-517. 